\title{
La famine de l'an 1316 en Flandre et dans les régions voisines
} H. Van Werveke

\section{Citer ce document / Cite this document :}

Van Werveke H. La famine de l'an 1316 en Flandre et dans les régions voisines. In: Revue du Nord, tome 41, n¹61, Janviermars 1959. pp. 5-14;

doi : https://doi.org/10.3406/rnord.1959.2324

https://www.persee.fr/doc/rnord_0035-2624_1959_num_41_161_2324

Fichier pdf généré le 07/04/2018 


\title{
LA FAMINE DE L'AN 1316 EN FLANDRE ET DANS LES REGIONS VOISINES
}

\author{
A M. J.-F. Verbruggen, \\ en témoignage de reconnaissance.
}

Les années 1315 à 1317 , on le sait, virent se succéder en Europe une suite de calamités. L'historien américain H. S. Lucas en a donné, il y aura bientôt une trentaine d'années, une vue d'ensemble richement documentée ${ }^{1}$. Il n'est guère, pour nos contrées, que les comptes communaux de Bruges - encore inédits - qui aient échappé à sa vigilance. La publication de leurs données sera pour nous l'occasion de reprendre le sujet, du moins en ce qui concerne la Flandre, le Brabant et la ville de Tournai. Nous espérons pouvoir apporter des précisions sur un certain nombre de points.

Le cadre géographique des événements décrits par Lucas - "des Pyrénées aux plaines de Russie, de l'Écosse à l'Italie" - est tellement vaste, que le tableau des calamités survenues durant ces trois années en devient quelque peu diffus. En nous bornant à les retracer dans les limites d'un territoire plus restreint, nous croyons pouvoir rendre plus distincts leur succession et leur enchaînement.

Commençons par indiquer, pour plus de clarté, les grandes lignes qui nous semblent devoir se dégager de notre exposé. Les conditions atmosphériques désastreuses de l'été de 1315 causèrent l'échec complet de la récolte. Dès les derniers mois de l'année les prix des céréales furent en hausse sensible. A partir du début de mai 1316 la situation devint catastrophique. Une fraction notable de la population, du moins dans les villes, périt de faim. La situation s'améliora graduellement après la rentrée de la nouvelle récolte. Des mesures appropriées prises à partir du printemps de 1317 par les autorités urbaines contribuèrent à empêcher un retour du désastre, en Flandre du moins. Le fléau dont souffrit la population de nos contrées fut donc essentiellement une crise de subsistance, limitée à l'année 1316. Il importe toutefois d'ajouter que dans sa suite une épidémie, dont la nature est incertaine, augmenta encore le nombre des victimes.

Les comptes communaux d'Ypres ont fourni à l'histoire des calamités, voici bientôt un siècle, des données statistiques d'une précision aussi parfaite qu'effrayante ${ }^{2}$ : le brief "de faire fouir les mors", figurant

1. Henry S. LucAs, The Great European Famine of 1315, 1316, and 1317 (Speculum V, 1930, pp. 343-377).

2. Comptes de la ville d'Y pres de 1267 à 1329, éd. G. des Marez et E. de Sagher, I (Bruxelles 1909), pp. 607-612. Ces donnés ont été uthisées entre autres par I. DiEGERICK, La peste à Ypres (1316 et 1317), (Annales de la Société historique, archéologique et littéraire de la ville d'Ypres et de l'ancienne West-Flandre, I, 1861, pp. 322-326), par H. PIRENNE, Les dénombrements de la population d'Ypres au $X V^{\ominus}$ siècle (1412-1506), (Vierteljahrschrift für Sozialund Wirtschaftsgeschichte, I, 1903, réimprimé dans : H. PIRENNE, Histoire économique de l'Occident médiéval, 1951, pp. 458-488, cité d'après cette réimpression), et par Lucas (v. note précédente). 
au compte de 1315-1316, nous apprend que du $1^{\text {er }}$ mai au 30 octobre de cette dernière année la ville indemnisa "frere Jake del ospital NostreDame pour faire porter et quillir les mors aval les rues " ${ }^{1}$; il apparaît que le nombre des morts comptabilisés, si l'on ose dire, de la sorte, semaine par semaine, s'élevait à $2.794^{2}$.

Pirenne estimait que ce texte était " sans doute le relevé le plus exact que l'on connaisse des ravages causés par une épidémie pendant le moyen âge " ${ }^{3}$. Lucas parle à ce propos de "the fortunate survival of an extraordinary document "4. Il se fait toutefois qu'un texte en tout comparable à celui d'Ypres repose encore à l'état inédit dans les archives d'une autre ville flamande. Le compte communal de Bruges relatif à l'année scabinale 1315-1316 contient en effet une rubrique formant, pour la même période de l'année 1316, un relevé qui est le pendant exact du "brief de faire fouir les mors $"$.

Nous avons réuni dans le tableau synoptique (page 7) les données statistiques de ces deux textes. Nous les avons reportées ensuite sur un graphique, afin de mieux faire ressortir les analogies et les divergences que présente l'évolution de la "mortalité " dans les deux villes.

On notera tout d'abord la concordance chronologique presque parfaite ${ }^{6}$. On ne pourra, en second lieu, manquer d'être frappé par la similitude des chiffres durant les premières semaines. Dans la suite, par contre, une différence notable s'accuse : à Bruges le nombre des morts diminue rapidement et de façon constante dès le milieu de juillet, tandis qu'à Ypres la courbe ne commence à fléchir que dans la première semaine d'août, et le fléau n'y semble conjuré que dans la troisième semaine de septembre.

Il ne s'agit pas seulement de mettre en regard les chiffres absolus.

1. Comptes d'Ypres, I, p. 607. A partir du 29 mai le libellé est conçu commc suit : " A frere Jake del ospital pour cheus qui ont asamblé les mors aval le ville".

2. Les chiffres figurant dans Diegerick (p. 326 : 3013 ) et dans Pirenne (p. 460 : * 2573 ) sont inexacts. Le chiffre de 2794 que nous donnons ici représente un minimum : il correspond aux données numériques fournies expressément par le compte de 1315-1316. Il faut observer que ce compte mentionne en outre des dépenses analogues pour les semaines

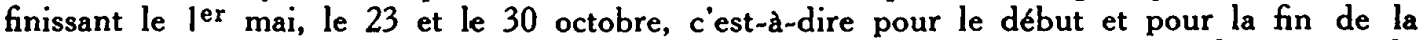
période de famine, mais sans que le nombre de morts soit spécifié. De même le compte de 1316-1317 (Comptes de la ville d'Y pres, II, Bruxelles 1913, p. 31) comporte des dépenses analogues pour l'exercice de cette année échevinale. Le nombre de morts n'est spécifié qu'une seule fois, à la date du 29 janvier 1317 : il ne s'élève qu'à onze. On peut donc dire sans crainte de trop s'aventurer que le chiffre de 2.794 représente à quelques dizaines près le nombre total des victimes tombant dans la catégorie définie dans le texte.

3. Comme nous le verrons aussitôt, il s'agit en réalité, non d'une épidémie, mais d'une famine.

4. P. 367.

5. Archives de la ville de Bruges. Compte $1315-1316$, fo $64 \mathrm{r}^{\circ}$ et $\mathrm{v}^{\circ}$. L'existence de cette rubrique nous a été très aimablement signalée par M. J.-F. Verbruggen, actuellement professeur à l'Université d'Élisabethville (Congo Belge), qui nous a en outre autorisé à nous servir de sa trouvaille. Nous tenons a lui exprimer ici toute notre reconnaissance.

Nous avions déjà mis en cuvre les données de ce texte, lorsque nous apprîmes que Madame Tits-Dieuaide, élève de notre collègue M. P. Bonenfant (Bruxelles), avait eu connaissance de ce texte au moment de préparer sa thèse de licence, relative aux famines du moyen âge. Madame Tits-Dieuaide, en même temps qu'elle nous assurait qu'elle ne comptait pas l'utiliser dans quelque publication, eut l'amabilité de dissiper nos derniers scrupules.

6. Notons quà Tournai également la "mortalité » commença à la date du ler mai : a L'an mil iijc xvj, le jour de may commencha uns grans mortoire à Tournay et moru bien en ung mois xij mile personnes." Croniques de Franche, d'Engleterre, de Flandres, de Lile et espécialment de Tournay, éd. A. Hocquet (Mons 1938), p. 36. Le chiffre de douze mille, cela va de soi, est sans aucune valeur comme donné historique. 


\begin{tabular}{|c|c|c|c|}
\hline \multicolumn{4}{|c|}{$\begin{array}{l}\text { Nombre de cadavres recueillis aux frais de la ville a Bruges } \\
\text { et à Ypres au cours de l'année } 1316\end{array}$} \\
\hline \multicolumn{2}{|c|}{ Semaine finissant $\mathrm{le}^{1}$} & Bruges & Ypres \\
\hline $\begin{aligned} & 30 \text { avril } \\
& 7 \text { mai } \\
& 14 \text { mai } \\
& 21 \text { mai } \\
& 28 \text { mai } \\
& 4 \text { juin } \\
& 11 \text { juin } \\
& 18 \text { juin } \\
& 25 \text { juin } \\
& 2 \text { juillet } \\
& 9 \text { juillet } \\
& 16 \text { juillet } \\
& 23 \text { juillet } \\
& 30 \text { juillet } \\
& 6 \text { août } \\
& 13 \text { août } \\
& 20 \text { août } \\
& 27 \text { août } \\
& 3 \text { septembre } \\
& 10 \text { septembre } \\
& 17 \text { septembre } \\
& 24 \text { septembre } \\
& 1 \text { er } \\
& 8 \text { octobre } \\
& 15 \text { octobre } \\
& 22 \text { octobre } \\
& 29 \text { octobre } \\
& \text { octobre }\end{aligned}$ & $\begin{aligned} & 1 \text { er } \text { mai } \\
& 8 \text { mai } \\
& 15 \text { mai } \\
& 22 \text { mai } \\
& 29 \text { mai } \\
& 5 \text { juin } \\
& 12 \text { juin } \\
& 19 \text { juin } \\
& 26 \text { juin } \\
& 3 \text { juillet } \\
& 10 \text { juillet } \\
& 17 \text { juillet } \\
& 24 \text { juillet } \\
& 31 \text { juillet } \\
& 7 \text { août } \\
& 14 \text { août } \\
& 21 \text { août } \\
& 28 \text { août } \\
& 4 \text { septembre } \\
& 11 \text { septembre } \\
& 18 \text { septembre } \\
& 25 \text { septembre } \\
& 2 \text { octobre } \\
& 9 \text { octobre } \\
& 16 \text { octobre } \\
& 23 \text { octobre } \\
& 30 \text { octobre }\end{aligned}$ & $\begin{array}{l}116 \\
165 \\
145 \\
128 \\
156 \\
135 \\
150 \\
136 \\
140 \\
135 \\
124 \\
95 \\
85 \\
59 \\
54 \\
30 \\
28 \\
19 \\
22 \\
16\end{array}$ & $\begin{array}{r}? \\
54 \\
? \\
173 \\
146 \\
101 \\
107 \\
157 \\
149 \\
155 \\
167 \\
158 \\
172 \\
190 \\
191 \\
130 \\
140 \\
148 \\
138 \\
124 \\
115 \\
37 \\
27 \\
15 \\
? \\
? \\
?\end{array}$ \\
\hline & & 1.938 & 2.794 \\
\hline
\end{tabular}

Il faut se demander aussi ce qu'ils représentent relativement au nombre d'habitants. Il n'est malheureusement pas possible d'atteindre sur ce point une grande précision. Sans doute peut-on admettre avec J. De Smet que Bruges comptait vers 1340 environ 35.000 habitants $^{2}$, et peut-on estimer qu'en 1316 le chiffre n'était pas sensiblement inférieur ou supérieur. Mais pour Ypres lincertitude est bien plus grande. On sait de source sûre qu'en 1412, après un siècle de décadence, la ville n'abritait plus que 11.000 habitants $^{3}$. En 1316 ils devaient être beaucoup plus nombreux.

1. A Bruges les dépenses de la semaine étaient portées en compte le vendredi, à Ypres le samedi. Nous nous sommes toutefois cru autorisé à mettre en regard semaine par semaine les données numériques relatives aux deux villes.

2. J. DE SMET, L'effectif des milices brugeoises et la population de la ville en 1340 (Revue belge de philologie et d'histoire, XII, 1933), p. 636.

3. H. VAN WERVEKE, De omvang van de leperse lakenproductie in de veertiende eeuw (Mededelingen van de Koninklijke Vlaamse Academie voor Wetenschappen, Letteren en Schone Kunsten van België, klasse der letteren, IX, 2. Anvers-Utrecht 1947, 32 pp. Avec résumé français), pp. 12-13. 
H. VAN WERVĖKE

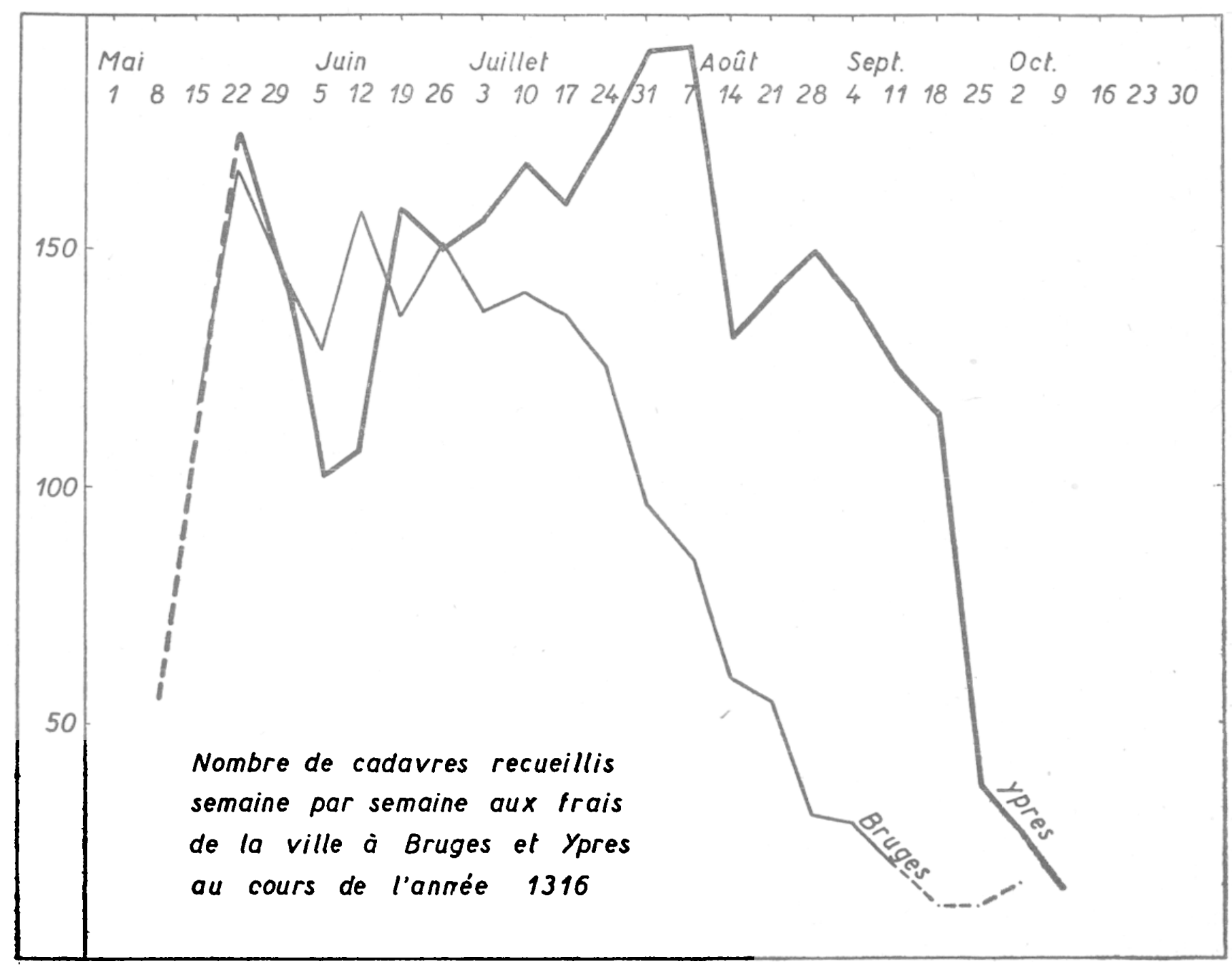

Mais de combien ? Je me suis risqué jadis à avancer un chiffre, que je répète aujourd'hui, faute de mieux $: 28.000^{1}$. Il peut toujours servir d'indication.

Si l'on voulait considerer un moment ces chiffres comme plus ou moins conformes à la réalité, les totaux des morts recueillis aux frais des villes, 1.938 pour Bruges et 2.794 pour Ypres, représenteraient respectivement $5,5 \%$ et $10 \%$ de la population. De toute façon le pourcentage doit avoir été sensiblement plus élevé à Ypres qu'à Bruges. Nous tenterons une explication plus loin.

Les ravages furent donc considérables, moins toutefois que ceux provoqués au milieu du siècle dans plusieurs pays d'Europe par la Peste Noire.

1. Pirenne, Dénombrements, p. 467. 
A quel fléau faut-il attribuer cette " mortalité " ? Diegerick et Pirenne songeaient précisément à la peste, et se prononçaient même assez catégoriquement à ce sujet. Des Marez et De Sagher se contentaient de parler d'“ épidémie " ${ }^{2}$.

Interrogeons les textes, et tout d'abord nos comptes communaux. Le texte yprois de 1315-1316 est plutôt muet à cet égard. On y parle de "tous les mors, qui sans lumiere seront enfoui ", de "tous cheaus qui morront sour le rue ", des "mors aval les rues" ${ }^{2}$. La rubrique du compte de 1316-1317 précise cependant qu'il s'agit des "cors des povres" ${ }^{3}$, indication que nous aurons l'occasion d'utiliser à l'instant.

Le compte de Bruges fournit quelques détails de même nature. Il y est question " de rassembler les morts en ville, dans les maisons et dans les rues, et d'enterrer ceux qui n'avaient pas d'“ amis " disposés à s'acquitter de cette tâche ${ }^{4}$. Si nous comprenons bien, il s'agit encore une fois de pauvres gens, dont les proches n'avaient pas les moyens d'assurer l'inhumation.

Nous manquons de données statistiques pour les villes autres que Bruges et Ypres. Mais les renseignements que nous possédons sur le Brabant et sur Tournai nous autorisent à dire que le même fléau y régna avec la même intensité. Ils proviennent de sources littéraires, qui s'expriment clairement sur sa nature. Ecoutons par exemple le chanoine Hocsem, chroniqueur liégeois, qui à ce moment séjournait à Louvain. Il nous parle de la cherté du blé et de la grande "mortalité " qui en résulta. Il nous raconte comment, deux ou trois fois par jour, une charrette venait prendre à l'hôpital de Louvain six à huit cadavres pour les transporter au nouveau cimetière aménagé aux abords de la ville ${ }^{5}$.

Mêmes scènes à Anvers, décrites avec plus de détails dans la chronique de Jan Boendale ( $=$ Jan de Klerk), rédigée en moyen néerlandais : pluies abondantes en 1315, récolte désastreuse, hausse exorbitante des prix dès les derniers mois de l'année. "Grande fut la détresse du peuple... Les cris et les pleurs des gens du peuple, qui se lamentaient couchés dans la rue, auraient dû attendrir jusqu'aux pierres. La faim faisait s'enfler leurs membres ". (Fdèmes de famine !) "Ils mouraient de misère sur place. On les jetait à soixante ou plus dans des fosses communes " ${ }^{6}$.

Van Velthem confirme les récits précédents pour l'ensemble du Brabant. Après avoir, lui aussi, parlé des intempéries de l'an 1315, il relate les bonds successifs des prix du blé, pour passer ensuite à la description de la "mortalité ". Il nous montre les pauvres tombant morts de faim, dans leurs maisons, dans les rues des villes, durant leurs pérégrinations à la campagne à la recherche de subsistances?. Dans toutes les villes du Brabant, ajoute-t-il, comme les cimetières existants ne pouvaient plus suffire, on en fit bénir de nouveaux, tant à l'extérieur qu'à

1. Comptes d'Ypres, I, p. 607, note 1. Lucas, p. 367, commence par parler très correctement de "famine", mais se sert également au cours de son exposé du mot " pestilence" (= maladie épidémique) : " until the week of October 9 when the pestilence abated " (p. 369), ce qui prête à confusion.

2. Comptes d'Ypres, 1, p. 607.

3. Ibid., II, p. 31 .

4. "Van den doden lieden te gaderne binder port, in husen ende achterstraten, ente delvene die gheene vriende hadden diere hand an slaen wilden."

5. La chronique de Jean de Hocsem, éd. G. Kurth (Bruxelles 1927), p. 162.

6. De Brabantsche Yeesten door Jan de Klerk, éd. J.-F. Willems, I (Bruxelles 1839), pp. 442-443.

7. Lodewijk van Velthem's voortzetting van den Spiegel Historiael, éd. H. Vander Linden, P. De Keyser et A. Van Loey, III (Bruxelles 1938), pp. 218-219, v. 1686-1697. 
l'intérieur des murs. Trois ou quatre fois par jour, des cadavres de pauvres morts de faim, entreposés provisoirement dans les hôpitaux, y étaient transportés par groupes de dix ${ }^{1}$.

Il n'en allait pas autrement à Tournai. Gilles le Muisit parle de la cherté des vivres, qui s'y faisait sentir dès l'an 1315, et qui prit une allure catastrophique à partir du début de mai 1316. La "mortalité " qui en résulta dépassa tout ce qu'on avait connu de mémoire d'homme. "Il y avait, ajoute-t-il, tant de pauvres mendiants qui se laissaient mourir dans les rues, sans même se soucier des immondices, que le magistrat de la cité dut se charger de faire évacuer leurs corps. On les transporta, afin de les y enterrer, en des endroits situés soit en deçà de l'Escaut, au Val de la Vigne et autres lieux, soit au delà du fleuve, aux Folais. Ceux qui étaient chargés de cette besogne étaient indemnisés au prorata du nombre des victimes transportées ${ }^{2}$.

On ne manquera pas d'être frappé par la concordance de ces témoignages, qui se complètent d'ailleurs admirablement. On ne peut en douter : la grande " mortalité " de 1316, à Bruges et à Ypres aussi bien qu'à Tournai et dans les villes brabançonnes, était due en ordre principal à une famine particulièrement grave, non à une épidémie.

On aimerait pouvoir reconstituer la courbe du prix des céréales. Plusieurs sources nous fournissent à ce sujet des données partielles. Elles nous permettent pour le moins d'affirmer que les prix montaient jusqu'à des niveaux 11,12 , même 24 supérieurs au niveau normal ${ }^{3}$.

On comprend que dans ces conditions il était impossible aux pauvres de se procurer des vivres. Les sources ne se font d'ailleurs pas faute de nous dire que les victimes de la famine se recrutaient exclusivement dans leurs rangs ${ }^{\star}$. Seules des personnes appartenant aux classes aisées, mais ne disposant pas de réserves alimentaires, étaient prêtes à payer ces prix exorbitants. Il y eut des propriétaires fonciers qui se ruinèrent pour éviter de mourir de faim ${ }^{5}$.

1. Van Velthem, III, p. 220, v. 1709-1727.

2. Chronique et Annales de Gilles le Muisit, éd. H. Lemaitre (Paris 1906), pp. 89-90. Ainsi que me le signale fort aimablement M. M.-A. Arnould, professeur a l'Université de Bruxelles, P. Rolland, dans la notice qu'il a consacrée a Tournai dans le Dictionnaire historique et géographique des communes du Hainaut, fasc. [ ${ }^{\mathrm{er}}$, pp. 5-7, observe que ces lieux-dits se trouvaient sur le territoire de la ville de Tournai. "Les Folais" existe encore, mais "le Val de la Vigne" a disparu.

3. Voici les principales données permettant de tracer des fragments de courbes de prix :

Van Velthem, III, pp. 216-217, v. 1629-1645 (Brabant) : le muid de Louvain (froment ?) se vendait $5 \mathrm{lb}$. du 1 er au 30 novembre (chiffre apparemment déja élevé); il monta à $7 \mathrm{lb}$. dès avant le 24 décembre 1315 , à $10 \mathrm{lb}$. avant le 11 avril 1316 , ensuite à $12 \mathrm{lb}$. et même à $16 \mathrm{lb}$. avant le 24 juin.

Gesta abbatum Trudonensium, MGH, SS, X, p. 416 (Hesbaye) : en 1316 le prix du muid de seigle était de 11 florins, en 1317 il était redescendu a 1 florin.

Annales Parchenses, MGH, SS, XVI, p. 608 (Louvain) : en 1316 le muid de seigle se vendait $24 \mathrm{lb}$; d dans la suite le prix redescendit a $1 \mathrm{lb}$.

Annales Fossenses, MGH, SS, IV, p. 33 (Fosses). En 1315 le muid d'épeautre valait 72 gros, en 131640 gros (a quel moment de l'année ?), en 1318 le prix redescendit a 6 gros.

4. Comptes d'Ypres, II, p. 31 : " cors des povres".

Van Velthem, III, p. 218, v. 1686 : « Ende som storven die arme lieden van honger.

De Klerk, I, p. 44, v. 814 : "Ende bleven van armoeden doot".

Gilles le Muisit, p. 90 : " tanta copia pauperum mendicantium"... "corpora pauperum sic morientium ".

5. Chronicon Jacobi Muevin (Corpus Chronicorum Flandriae, éd. J.-J. de Smet, II, Bruxelles 1841), p. 457 : "plurimis haereditates suas propter inopiam victualium vendentibus, et ob hoc ad summam paupertatem devolutis". 
Est-ce à dire que le pays manquait complètement de vivres ? Hocsem le nie formellement : "Les greniers regorgeaient de blé, dit-il, mais on préférait l'exporter vers les régions côtières" (Flandre, Zélande, Hollande ?), "où la cherté était plus grande encore " (qu'en Brabant) ${ }^{1}$. Si étonnante que cette assertion puisse paraître à première vue, le fait est normal. De tout temps les propriétaires fonciers du moyen âge qui avaient des revenus en nature, et sans doute encore d'autres catégories de gens aisés, veillaient à profiter des années de bonne récolte, pour se constituer des réserves leur permettant d'affronter les années de crise alimentaire ${ }^{2}$. La famine de 1316 ne les affecta pas. Bien plus, elle fut pour beaucoup d'entre eux l'occasion de réaliser des profits exceptionnels.

Avant d'en finir avec la famine proprement dite, il nous faut revenir sur les données de notre graphique. Il nous reste en effet à expliquer pourquoi le nombre des victimes fut relativement plus élevé à Ypres qu'à Bruges, même au cours des premières semaines, et pourquoi le mal fut enrayé plus tôt à Bruges qu'à Ypres. Il est assez probable que la réponse à la première question doit être cherchée dans le fait qu'Ypres était plus foncièrement un centre industriel, et doit par conséquent avoir compté un plus grand nombre d'ouvriers salariés, donc de gens pauvres. Pour le second point il faut sans doute songer à cette circonstance que Bruges, disposant des ports du $Z$ win, pouvait plus facilement se ravitailler par mer, pouvait même mettre la main, à son profit, sur toutes les cargaisons amenées dans l'estuaire. L'importance de cette situation privilégiée apparaîtra clairement par la description des mesures prises par le magistrat brugeois en 1317.

S'il est vrai que la famine fut la principale cause directe de la " mortalité ", il n'en reste pas moins qu'elle fut suivie d'une épidémie, du moins dans certaines villes. Les comptes de Bruges et d'Ypres sont muets à cet égard. Les textes brabançons et tournaisiens par contre fournissent des données intéressantes, bien qu'insuffisamment précises.

Boendale (Anvers) distingue nettement une succession chronologique de trois sortes de maux : les pluies excessives de 1315, la famine qui en fut la conséquence, et enfin l'épidémie. Au sujet de cette dernière, deux détails à retenir : le nombre relativement élevé de victimes, et le fait qu'elle frappait, à la différence de la famine, aussi bien les riches que les pauvres ${ }^{3}$. Van Velthem (Brabant) insiste également sur ce dernier point ${ }^{4}$. Il ajoute que les malades étaient atteints de fièvre, ce qui fut cause que le mal fut dénommé l' incommodité ardente ${ }^{5}$.

Gilles le Muisit (Tournai) qui, comme l'on sait, dicta sa chronique

1. Hocsem, p. 162. - L'explication du fait que la cherté était plus grande dans les régions côtières est donnée par le même auteur quelques lignes plus loin : $\alpha$ et circa mare propter inundationem defecit annona".

2. Qu'on se reporte au récit que donne Galbert de Bruges de la famine de 1124-1125 en Flandre. Les faits, sans doute, sont antérieurs de deux siècles, mais les conditions générales ne changeront guère dans l'intervalle. Galbert raconte comment le comte de Flandre Charles le Bon, au plus fort de la famine, fait distribuer du pain à des centaines de pauvres, utilisant les réserves de blé entassées dans ses châteaux. C'est dire que ces réserves étaient plus que suffisantes pour assurer la subsistance de la cour et de l'administration comtales. (Histoire du meurtre de Charles le Bon, Comte de Flandre (1127-1128) par Galbert de Bruges, éd. H. Pirenne. Paris 1891, p. 6). En 1316 l'abbaye de Saint-Trond fut en mesure d'agir de même envers les pauvres (Gesta abbatum Sancti Trudonis, MGH, SS, X, p. 416). On pourrait facilement multiplier ces exemples.

3. De Klerk, I, p. 443, v. 821-833.

4. Van Velthem, III, p. 219, v. 1698-170I.

5. " thete ongemac ". Van Velthem, III, p. 219, v. 1704. 
une trentaine d'années après les événements dont il est question ici, ne fait pas comme les deux auteurs précédents la distinction chronologique entre la famine et l'épidémie. Mais alors qu'il parle uniquement de pauvres à propos des enterrements aux frais de la ville, conséquence de la famine, il insiste sur le fait que l'" aer corruptus" - allusion claire à une épidémie - causa la mort de "potentes" aussi bien que de "medii " et de "mediocres", de "divites" aussi bien que de "pauperes" ".

On peut, comme nous le disions, conclure sur ce point que la famine fut suivie d'une épidémie. On peut y ajouter, sans crainte de trop s'avancer, qu'elle favorisa celle-ci, en débilitant la population, et même qu'elle en fut la cause, en ce sens que les pauvres, poussés par la faim à se servir d'une nourriture immonde, finissaient par négliger les règles les plus élémentaires de l'hygiène.

Les données des chroniqueurs sont malheureusement trop vagues pour que l'on puisse se prononcer sur la nature de la maladie ${ }^{2}$. Il faut écarter l'hypothèse d'une épidémie de peste. Van Velthem, dans ce cas, aurait sans doute mentionné un symptôme plus spécifique que la fièvre. Nous sommes d'ailleurs mieux renseignés que nos prédécesseurs au sujet de la diffusion de ce mal : nous savons aujourd'hui qu'il est fort douteux que la peste (bubonique) ait fait sa réapparition en Europe avant le milieu du XIVe siècle (la Peste Noire) ${ }^{3}$.

\section{II}

En 1316 le magistrat des villes fut apparemment pris au dépourvu par l'ampleur de la catastrophe. En 1317, instruit par l'expérience, il se tint sur ses gardes. La récolte de 1316, sans nul doute, l'emporta de beaucoup sur celle de 1315. Certes, au printemps de 1317, on pouvait encore se demander si elle suffirait entièrement aux besoins de la consommation. Du moins les échevins de Bruges prirent-ils des mesures pour assurer la "soudure" avec la récolte de 1317. Le compte spécial de ccttc politique de ravitaillement nous a été conservé, mais, à notre connaissance. il n'a pas jusqu'à présent attiré l'attention des historiens ${ }^{4}$.

La ville acheta à partir du mois d'avril 1317 des stocks considérables de blé à différents marchands ${ }^{5}$, dont certains peuvent être identifiés

1. Gilles le Muisit, blz. 89-90. - Signalons ici l'intéressant article de P. RuElLE, Un testament tournaisien de 1316 (Mémoires et Publications de la Société des Sciences, des Arts et des Lettres du Hainaut, $72^{\mathrm{e}}$ volume, pp. 51-73). Le testament fut rédigé en juillet 1316, sous l'impression de la "mortalité" qui sévit a Tournai à ce moment-là.

2. L'identification proposée par les éditeurs, à la suite d'une communication du docteur A.-F.-C. Van Schevensteen, nous semble insuffisamment fondée.

3. La peste (bubonique) qui sévit à Constantinople à l'époque de Justinien semble s'être répandue par la suite en Europe occidentale, et y être réapparue à intervalles pendant plusieurs dizaines d'années, peut-être même plusieurs siècles. Certains textes encore plus tardifs semblent également faire allusion à cette maladie. Ils ont été relevés dans l'ouvrage de G. STICKER, Die Pest, I (Giessen 1908), pp. 36-41. Ils demanderaient à faire l'objet d'une étude critique. Il semble en tout cas fort peu probable que l'Europe occidentale ait connu la peste durant le demi-siècle qui précède l'irruption de la Peste Noire (1347).

4. Archives de la ville de Bruges. Compte de 1317, intitulé : "Dit es de rekeninghe van den corne Jans bachter Kist ende Will. van Ansame van den jare $\mathrm{M}^{\circ} \mathrm{CCC}{ }^{\circ} \mathrm{XVII}{ }^{\circ}$."

5. Ce sont :

1. "Siere Asselyn Griec ". Peut-être un Ecossais. Cf. L. Gilliodts-van Severen, Cartulaire de l'ancienne Estaple de Bruges (Bruges 1904-06), II, pp. 249-250, no 1219 : "Jan Grig van Abberdan in Schotland" (1485).

2. "Siere Nicholas" (?).

3 et 4. "Alafonse Lorem" et "siere Bonote Lorem". Sans doute des Catalans. En 1317 les Catalans étaient les seuls Espagnols à séjourner à Bruges.

5. "Siere Anthone de Mare :. Mentionné deux fois. Certainement un Génois. R. Doemaerd, 
comme originaires de pays méditerranéens. La marchandise, avant l'acquisition, était entreposée soit à Damme, soit chez des hosteliers brugeois. Le total des achats auquel procéda le magistrat s'éleva à $11.785,75$ heu de froment, soit, à raison de $1 \mathrm{hl}$. 6661 par heu, une quantité de $19.636 \mathrm{hl}$. Si l'on s'en tient au chiffre de 35.000 habitants, cela représente un peu plus de 56 l. par tête d'habitant. Il faut y ajouter 500 heu de seigle, soit $833 \mathrm{hl}$. ou 2.4 l. par habitant. Si l'on considère que ces céréales furent mises à la disposition de la consommation urbaine durant une période qui ne dépassa pas trois mois et demi, on arrive à la conclusion qu'à cette époque critique de l'année la population doit avoir vécu en ordre principal de ces stocks réunis par les soins du magistrat. Cela ne signifie évidemment pas que, sans ces mesures, la famine aurait régné à nouveau. Mais il est fort probable qu'en empêchant l'accaparement du blé par les marchands professionnels et en prenant elles-mêmes en main la distribution des vivres, les autorités urbaines purent assurer à la population un ravitaillement à bien meilleur compte que sur le marché libre.

Le premier stock fut réparti entre les boulangers à partir du 11 avril 1317, le dernier fut liquidé le 2 août. La ville avait acheté le blé aux mar chands à des prix qui variaient entre $8 \mathrm{~s}$. $3 \mathrm{~d}$. st. et $10 \mathrm{~s}$. $9 \mathrm{~d}$. st. le heu. Elle les revendit aux boulangers à un prix à peu près uniforme, calculé de telle sorte que l'opération se solda finalement sans laisser un déficit trop élevé ${ }^{1}$. Les dépenses s'élevèrent au total à $78.306 \mathrm{lb}$. de payement, les recettes à $73.985 \mathrm{lb}$. Remarquons, pour que le lecteur puisse se faire une idée de l'importance de ces sommes, qu'elles étaient du même ordre de grandeur que les dépenses et recettes ordinaires de la ville de Bruges.

On peut admettre qu'au 2 août, lorsque la vente aux boulangers prit fin, l'éventualité d'une famine était conjurée. Peut-être les quantités distribuées pouvaient-elles suppléer aux besoins de la consommation jusqu'à la rentrée de la nouvelle récolte, et sans doute celle-ci s'annonçait-elle comme normale. Toujours est-il qu'à ce moment-là les autorités urbaines furent assaillies par des soucis d'un ordre différent. On les vit prendre des mesures qui semblent aller à l'encontre du but visé jusque là.

Sur le dernier stock acquis par la ville, 2.418,5 heu furent distribués aux boulangers. Les 440 heu restants reçurent une autre destination : 118 heu furent vendus, à raison de $6 \mathrm{lb}$. $15 \mathrm{~s}$. le heu, à un consortium de marchands de blé ${ }^{2}$, et 322 heu furent cédés, moyennant $9 \mathrm{lb}$. le heu,

Les relations commerciales entre Gênes, la Belgique et l'Outremont (Bruxelles-Rome 1941, III, p. 1088, no 1792 : "Anthonius de Mari" - "civis Janue" - "constitue Lomellinus et Manfredus de Mari ses mandataires certains en France, aux foires de Champagne et de Flandre et à Bruges " (30 avril 1313).

6. "Siere Georges ". Peut-être un Vénitien. Il y a en 1469 à Bruges un François George, consul de la nation de Venise. Gilliodts, Estaple, II, p. 187, no 1129.

7. "Siere Ogier Pervoisyn". Certainement un Génois. On rencontre en 1267 à Gênes un "Ogerius Pilavicenus", qui n'est évidemment pas notre personnage, mais qui pourrait être son grand-père. Doemaerd, Relations, III, p. 694, n 1251.

8. "Siere Dominike Scortse Fighe". (?).

Je remercie M. J. Maréchal, qui a bien voulu maider à identifier certains de ces personnages.

1. Le premier stock fut vendu aux boulangers à raison de $5 \mathrm{lb}$. $14 \mathrm{~s} .$, les autres à raison de $6 \mathrm{lb}$. le heu.

2. "...den coerenbiters Willeme bachter Kist ende sinen gheselschepe". Les corenbiters ou marchands de blé, qui en temps normal s'acquittaient d'úne fonction économique utile en assurant l'approvisionnement de la population, se muaient en temps de disette en accapareurs, prêts à exploiter à leur profit la détresse de la masse. Le fait que la ville cède une partie du blé aux marchands prouve précisément qu'il n'y avait plus de disette à craindre. 
à des gens qualifiés de buskynbakers ${ }^{1}$. Comme le prix payé par les bou langers était normalement de $6 \mathrm{lb}$., il est facile de calculer que cette double opération valait à la ville une recette supplémentaire de $1.054 \mathrm{lb}$. $10 \mathrm{~s}$., réduisant d'autant le déficit, qui finit par se chiffrer à $4.321 \mathrm{lb} .{ }^{2}$. Sans doute était-ce le souci de ménager le budget ordinaire, sur lequel cette somme devait en fin de compte être imputée, qui amena les échevins et les trésoriers à prendre in extremis cette mesure quelque peu déconcertante.

La famine que nous avons décrite, causée par la mauvaise récolte de 1315, due elle-même aux pluies extrêmement abondantes dont souffrit l'Europe toute entière en cette année, est l'une des dernières catastrophes de cette nature et de cette ampleur qui aient affecté notre continent dans son ensemble. Elle est en même temps la première pour laquelle on dispose de données statistiques précises et dignes de foi. Dans les Pays-Bas méridionaux elle resta circonscrite à l'année 1316, les derniers mois de 1315 ne comportant que les premières phases de la hausse des prix, et l'année 1317 marquant la transition vers une situation normale, transition favorisée il est vrai par l'intervention des autorités urbaines. C'est aussi la première fois que l'on peut suivre de près, du moins dans nos régions, l'application d'une politique de ce genre.

Quant aux maladies, leur rôle dans la catastrophe fut secondaire. Il est d'ailleurs fort possible que, si des épidémies sont signalées un peu partout en Europe au cours de ces années fatales, elles étaient le fait de maladies de nature diverse, se greffant chacune de son côté sur l'état de sous-alimentation qui affectait la population de l'Occident toute entière.

\author{
(Université, Gand).
}

H. VAN WERVEKE.

1. Le mot buskynbakers n'a pas été relevé par les lexiques de moyen néerlandais. D'après le contexte il ne peut s'agir que de personnes employant de la farine pour la production d'articles de boulangerie plus fins que le pain ordinaire.

2. Il est certain que Bruges ne fut pas la seule ville de Flandre à prendre au printemps de 1317 des mesures en vue du ravitaillement de sa population. Le compte de la ville de Gand de 1316-1317 nous apprend que du 9 au 14 avril 1317 deux échevins gantois se rendirent à un parlement, tenu au château comtal de Male, près de Bruges, où le " pays de Flandre "était réuni ; ils devaient y traiter de la part revenant aux Gantois dans un stock arrivé récemment ("omme ons deel vanden corne datter commen was"). Un autre passage du même compte nous renseigne sur le fait que vers le 30 avril du blé appartenant aux Gantois (celui-là même peut-être dont il vient d'être question) avait été arrêté à Damme, sur le canal de la Lieve, par ceux du Franc de Bruges. On ignore la suite de l'affaire (Comptes de la ville et des baillis de Gand, 1280-1336, éd. J. Vuylstèke, Gand 1900, pp. 115, 1.33, et 129, 1. 3-4).

Ypres était également représenté par deux délégués au parlement tenu vers le $9-14$ avril à Male. Comptes de la ville d'Ypres, II, p. 14, 1. 21-22. 